This item was submitted to Loughborough's Research Repository by the author.

Items in Figshare are protected by copyright, with all rights reserved, unless otherwise indicated.

\title{
Impact of surface discharge plasmas on performance of a metallized film capacitor
}

PLEASE CITE THE PUBLISHED VERSION

PUBLISHER

(C) American Institute of Physics

\section{VERSION}

VoR (Version of Record)

\section{LICENCE}

CC BY-NC-ND 4.0

\section{REPOSITORY RECORD}

Kong, Michael G., and J.P. Lee. 2019. "Impact of Surface Discharge Plasmas on Performance of a Metallized Film Capacitor”. figshare. https://hdl.handle.net/2134/5166. 
This item was submitted to Loughborough's Institutional Repository (https://dspace.lboro.ac.uk/) by the author and is made available under the following Creative Commons Licence conditions.

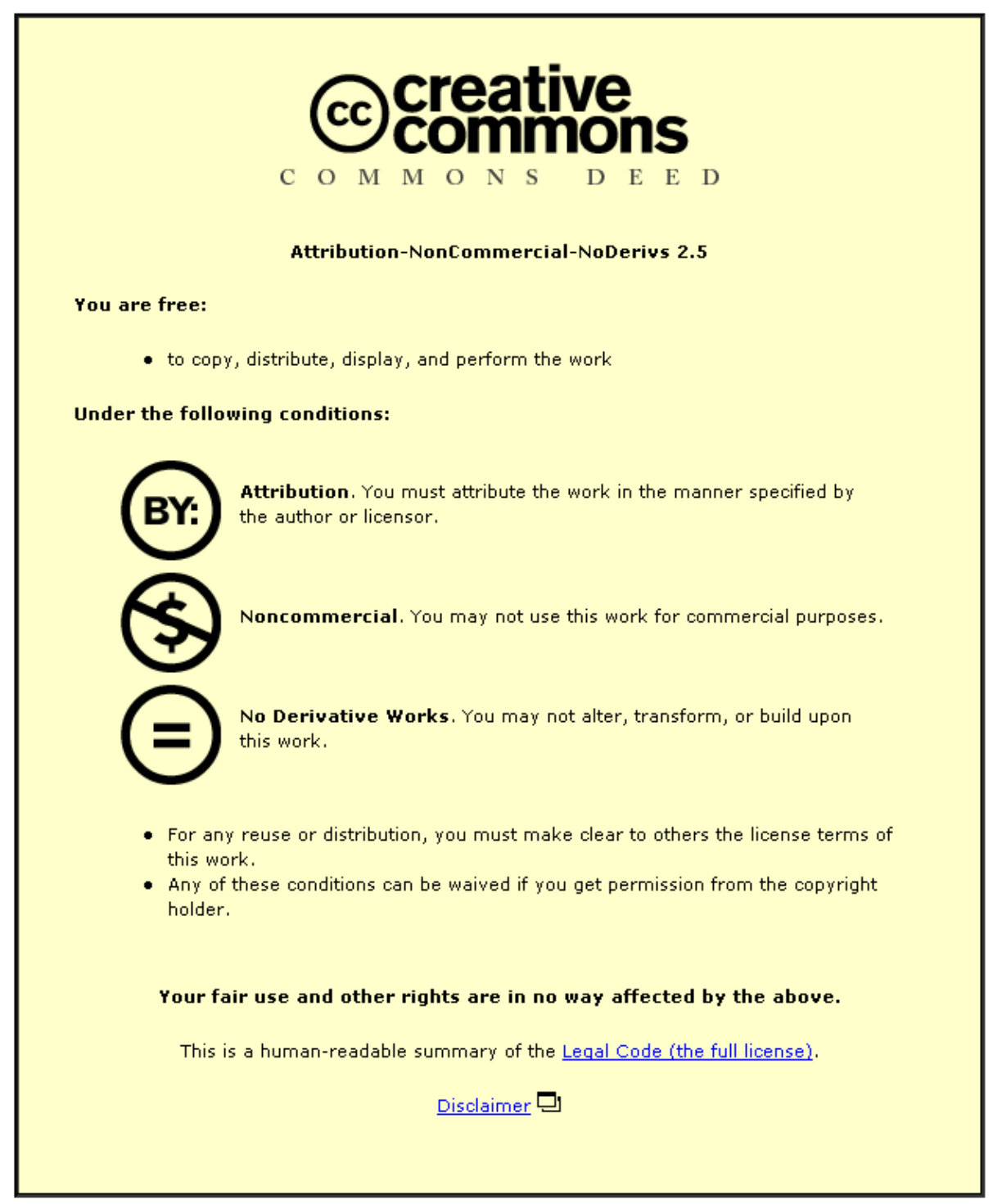

For the full text of this licence, please go to: http://creativecommons.org/licenses/by-nc-nd/2.5/ 


\title{
Impact of surface discharge plasmas on performance of a metallized film capacitor
}

\author{
M. G. Kong ${ }^{\text {a) }}$ and Y. P. Lee \\ Department of Electronic and Electrical Engineering, Loughborough University, Leicestershire LE11 $3 T U$, \\ United Kingdom
}

(Received 12 October 2000; accepted for publication 6 June 2001)

\begin{abstract}
Surface breakdown discharges are one probable failure mechanism of metallized polymeric film capacitors used in power systems, traction drives, and other technological applications. To assess whether surface breakdown discharges may undergo considerable elongation on the electrode surface to affect significantly capacitor performance, an equivalent electric circuit model is developed for metallized polymer film capacitors under the thermal equilibrium condition. With the aid of a surface field gradient mechanism, propagation of surface plasmas is studied and the necessary condition for their possible elongation is obtained. Numerical examples of a metallized film capacitor are used to demonstrate that surface breakdown plasmas and their elongation are unlikely to affect capacitor performance in a significant fashion. Then the generic problem of plasma propagation is restudied under thermally nonequilibrium conditions. Based on a heat conduction formulation in the one-dimensional limit, a temperature gradient mechanism is proposed to explain the possible elongation of breakdown plasmas on an electrode surface. Numerical examples are again used to deduce that thermally nonequilibrium surface plasmas are unlikely to evolve into catastrophic flashover arcs to fail film capacitors. (c) 2001 American Institute of Physics.
\end{abstract}

[DOI: 10.1063/1.1389072]

\section{INTRODUCTION}

There has been much interest in metallized polymeric film capacitors, fueled by their widespread applications in power systems, traction drives, laser energization, and spacebased technologies. ${ }^{1,2}$ Through a largely experimental approach, intense research and development efforts have been focused on many key aspects of metallized film capacitors, for instance, the properties of polymeric films and their degradation behaviors under mechanical stresses, ${ }^{3}$ electrical stresses (dc, ac, and pulsed), ${ }^{3-5}$ and thermal stresses. ${ }^{6}$ These efforts are largely responsible for some 30-fold increase in energy density achieved in high-voltage-metallized film capacitors over the past 20 years. ${ }^{6}$

One of the main thrusts in the field is to further increase energy density with increasingly compact capacitor designs. As higher and higher energy density is achieved, the electric field within film capacitors becomes larger and larger. Inevitably, this leads to a significantly increased probability for ignition of breakdowns and breakdown discharges. There is, therefore, an important need to capture a thorough understanding of breakdown phenomena within film capacitors. A more pressing issue for practical film capacitors, however, is to answer whether high-field-induced breakdowns may implicate significantly capacitor performance of existing and future designs, and if so, how key system parameters can be adjusted to minimize such adverse impact. One key challenge to understand breakdown phenomena in film capacitors is the difficulty to perform in situ diagnostics on tightly packed film capacitors, which are themselves packaged into

\footnotetext{
${ }^{a)}$ Electronic mail: m.g.kong@lboro.ac.uk
}

sealed units. External measurements can, at best, indicate device-specific impact of capacitor breakdowns and their discharge plasmas, but are not effective in capturing the underlying physics that can be applied elsewhere more generically. As a result, the impact of breakdown discharges on capacitor performance is usually assessed empirically through a large number of accelerated life tests and subsequent visual breakdown damage analyses on aged film capacitors. This is an expensive and time-consuming approach, but can be greatly mitigated if a viable theoretical model is developed. Given that the significant advance of the film capacitor technology over the past 20 years has been largely based on an extensive exploitation of wide-ranging experimental techniques, viable theoretical models are, by comparison, more likely to bring the next breakthrough in the metallized film capacitor technology.

There are at least two different types of breakdown discharges in film capacitors, namely, flashover arcs on the electrode surface and puncture plasmas through multiple film layers. While there are very few theoretical treatments of breakdown discharges in film capacitors, puncture discharges and their effects have been attempted with a plasma dynamic model. ${ }^{7}$ In contrast, surface breakdowns and their impact on capacitor performance have received much less attention. ${ }^{8}$ It has been known that once ignited surface discharges can be sustained at an electric field several magnitudes below the breakdown strength of the insulating medium used. ${ }^{8,9}$ Therefore, at relatively low surface electric field, surface plasmas may elongate on the electrode surface and can potentially develop into catastrophic flashover arcs to fail film capacitors. It is, therefore, important to assess whether surface dis- 
charges induced in film capacitors may affect significantly capacitor performance.

A thorough knowledge of surface discharges requires an understanding of their ignition and that of their subsequent evolution. It is known that surface discharges in film capacitors are usually triggered by localized surface field intensification, which are often caused by surface defects resulting from various manufacturing processes. Ignition of these surface discharges and their spatial distribution are, therefore, likely to be statistical, reflecting an inevitable statistical fluctuation in the quality of the polymeric film and its electrode coating. It should be noted, however, that an understanding of such a statistical nature of surface discharges do not necessarily indicate their eventual impact on capacitor performance, as the latter depends crucially on surface electric field. In our experiments to induce breakdowns on the surface of an air-insulated single-metallized polymer film, the induced surface discharges were found to last, typically, a few picoseconds at up to $1 \%$ of the breakdown field strength in air. Also, these mild discharges did not affect the surface condition of the film in any significant way. On the other hand, the presence of a sizable surface electric field can drive an initially small surface discharge to undergo considerable elongation on the electrode surface. Thus, to answer whether surface breakdowns may affect significantly the reliability and lifetime of a film capacitor, it is much more important and relevant to understand whether and how a surface plasma may elongate rather than whether and how it is ignited in the first place.

In this article, we study the propagation of surface breakdown plasmas on the capacitor electrode, assuming that they have already been ignited, with our emphasis on the condition under which they may undergo an unrestricted elongation. This is based on our recent work on this subject, ${ }^{8}$ which has described the development of a relevant mathematical foundation but has not answered the question of whether surface plasma may cause catastrophic failure in metallized film capacitors. In Sec. II, we review the mathematical framework of our analysis of propagation and elongation of surface plasmas that are at a thermal equilibrium with their surrounding capacitor structure. A field gradient mechanism is then used to explain the condition for plasma elongation, and an in-depth discussion is provided to highlight the likely impact of the field gradient mechanism on plasma elongation. It is shown that for practical film capacitors it is unlikely for the field gradient mechanism to drive a sustained elongation of surface discharges. Then, in Sec. III, plasma propagation is restudied under thermally nonequilibrium conditions and a temperature gradient mechanism is proposed to assess whether sustained plasma elongation may be possible. With a more accurate estimate of the likely electricfield induced on the electrode coating surface than that used in Ref. 8, numerical examples are used to illustrate quantitatively the most likely impact of surface discharge plasmas and their possible elongation upon performance of metallized film capacitors. Again, substantial plasma elongation is found unlikely under the nonthermal equilibrium condition. Finally, in Sec. IV, conclusions drawn in this study are summarized.

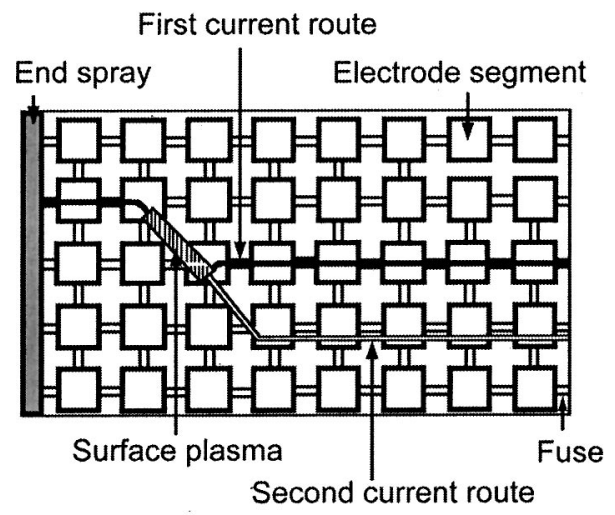

(a)

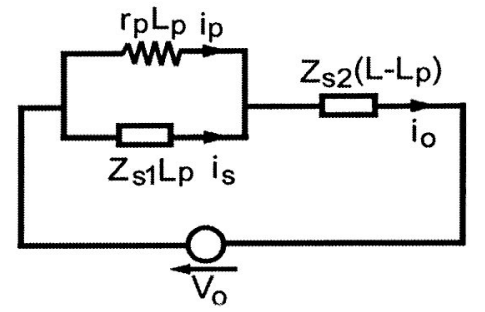

(b)

FIG. 1. (a) Surface discharge and its current routes on the electrode surface and (b) its equivalent circuit.

\section{IMPACT OF SURFACE DISCHARGES UNDER THE THERMAL EQUILIBRIUM CONDITION}

For practical film capacitors, there are inevitably defects on the electrode surface resulting from manufacturing processes of the polymeric film, its metallization, and its eventual packaging into the capacitor casing. These surface defects enhance surface electric-field locally, and as such, increase significantly the likelihood to ignite localized surface breakdowns. Once a breakdown discharge is ignited from a surface defect site, it may propagate on the electrode surface of a film capacitor. Suppose the discharge plasma is sufficiently cold to be in a thermal equilibrium with its surrounding capacitor structure, its propagation on the electrode surface will be predominately controlled electrically through the surface electric field. As a result, plasma propagation on the electrode surface can, under the thermal equilibrium condition, be described by electrical characterization only.

The electrode coating on the surface of a polymer film is often patterned with, usually, identical segments networked together via conducting fuse links between them, as illustrated in Fig. 1. During a time-dependent application of an external voltage (e.g., external switching), the film capacitor structure is charged or discharged from the film edge (nearer to the capacitor terminals) towards its central area, thus producing temporally a surface potential variation across the electrode coating length. ${ }^{10}$ The resulting surface electrical field induces a surface current to flow on the electrode surface, and usually this surface current is a conduction current along fuse-linked, thus electrically conductive, electrode segments, although a displacement surface current is also possible through gaps between adjacent electrode segments. ${ }^{10}$ When a discharge plasma is induced by a local breakdown 
on a defect site on the electrode surface, such surface current flows through the plasma column to support the latter electrically, as shown in Fig. 1. The plasma supporting electric energy is likely to be fed via the original defect site, at least initially, and for the simplicity of our discussion we assume that the surface discharge has a stationary root at the defect site and its other root moves on the electrode surface. These two current roots are the termination points of the plasma column on the electrode surface, and they connect to a nonplasma, but fuse-linked, section of the electrode surface, as shown in Fig. 1. Under the influence of the surface electric field, the current leaving the moving root of the plasma may flow along two possible routes on the segmented electrode surface. As illustrated in Fig. 1, the first route is through successive electrode segments linked to each other by unbroken fuses, whereas the second is through unconnected electrode segments with either separation gaps or broken fuses between them. If we model electrode segments with surface resistors and separation gaps (or broken fuses) with surface capacitors, the discharge current may be considered to flow through a series of resistors and capacitors along the current path unoccupied by the plasma. In other words, the current route of the nonplasma section may be described by means of an equivalent circuit consisting of surface resistors and surface capacitors connected in series, no matter whether the plasma-driving current takes the first or the second route. It should be noted that the representation of the plasma using a lumped resistor is likely to underestimate the nonlinear impact of its dynamics. Nevertheless, the resulting equivalent circuit-based model is effective in unraveling the basic plasma effects in many practical application areas such as outdoor insulators. ${ }^{9,11}$

As a one-dimensional approximation, the surface discharge is assumed to be a uniform cylindrical column having a length of $L_{p}$ and a cross-sectional area of $A_{p}$. We further assume that it can be modeled approximately by a resistor with resistivity $\rho_{p}$ and resistance of

$$
R_{p}=\rho_{p} L_{p} / A_{p}=r_{p} L_{p},
$$

where $r_{p}$ is the plasma resistance per unit length. The presence of the surface plasma alters the equivalent impedance of the electrode surface along the current route by effectively adding a parallel resistor $R_{p}$ to the surface impedance, $z_{s 1} L_{p}$, of the electrode area underneath the surface plasma of which $z_{s 1}$ is the unit length impedance. The current path can then be described approximately by the equivalent circuit in Fig. 1(b), where $L$ is the overall length of the current route and $z_{s 2}$ is the unit length impedance of the electrode surface area not covered by the surface plasma.

If $r_{p}>\left|z_{s 1}\right|$, a small fraction of the total surface current, $i_{0}$, flows through the surface plasma, and so the plasma propagation is driven by only a small part of the total energy of the surface field. This plasma-driving electric energy reduces further, as the plasma elongates to become longer and more resistive [see Eq. (1)]. Thus, even for the cases where the surface plasma does elongate initially, it is unlikely for the plasma to draw sufficient electric energy subsequently to evolve into a catastrophic flashover arc. It is, therefore, reasonable to assume that $r_{p}>\left|z_{s 1}\right|$ is the condition under which

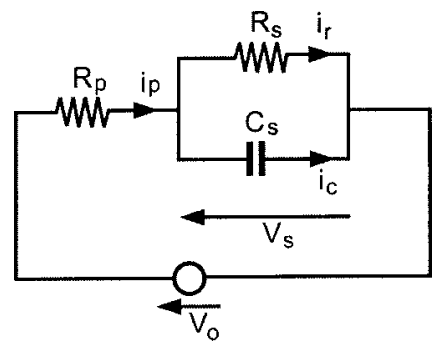

FIG. 2. Simplified equivalent circuit model of surface discharge plasmas.

the surface discharge can, at most, develop into a short-lived and insignificantly elongated plasma before it extinguishes. If $r_{p}<\left|z_{s 1}\right|$, on the other hand, the plasma can draw sizable current, and so gain a sufficient amount of electric energy for its subsequent propagation away from its stationary root. If we further assume that $r_{p} \ll\left|z_{s 1}\right|$, the plasma can then draw most of the electric energy supplied to undergo a significant elongation. This suggests that $r_{p} \ll\left|z_{s 1}\right|$ is the condition under which a surface discharge has a high probability of undergoing considerable elongation.

Under the condition of $r_{p} \ll\left|z_{s 1}\right|, z_{s 1} L_{p}$ may be ignored in the equivalent circuit of Fig. 1(b), and this corresponds to the most severe situation where the plasma is driven by the maximum possible electric energy of the surface field. The plasma column is now connected in series directly to $z_{s 2}(L$ $-L_{p}$ ), the surface impedance along the current path not covered by the plasma. The equivalent circuit in Fig. 1, therefore, reduces to that in Fig. 2. It is worth mentioning that the equivalent circuit in Fig. 2 is similar to that proposed recently for outdoor insulators, ${ }^{11}$ albeit the difference in the context of application areas and in the physical meaning of individual circuit components.

There are two components in the surface impedance of the nonplasma section, namely, a lumped surface resistor, $R_{s}$, representing the total resistance of electrode segments, and a lumped surface capacitor, $C_{s}$, representing the total capacitance of separation gaps, both over the length of ( $L$ $\left.-L_{p}\right)$. Let $\rho_{s}$ be the resistivity of electrode material, $r_{s}$ its unit length resistance, $\varepsilon_{r}$ the relative permittivity of the gap material, and $A_{s}$ the cross-sectional area of the gap, we have

$$
\begin{aligned}
& R_{s}=\rho_{s}\left(L-L_{p}\right) / A_{s}=r_{s}\left(L-L_{p}\right), \\
& C_{s}=\varepsilon_{0} \varepsilon_{r} A_{s} / g,
\end{aligned}
$$

where $g$ is the accumulated spacing between all adjacent electrode segments along the nonplasma current route. The total impedance of the equivalent circuit is, therefore,

$$
Z_{T}=R_{p}+R_{s} /\left(1+j \omega R_{s} C_{s}\right),
$$

with its square modulus as a function of $L_{p}$ given by

$$
\left|Z_{T}\right|^{2}=v L_{p}^{2}+\frac{\rho_{s}^{2} L^{2}}{a A_{s}^{2}}+\frac{2 \rho_{s}}{a A_{s}} \frac{\rho_{p}}{A_{p}}-\frac{\rho_{s}}{A_{s}} L L_{p} .
$$

Here,

$$
a=1+\omega^{2} R_{s}^{2} C_{s}^{2},
$$




$$
v=\frac{\rho_{p}^{2}}{A_{p}^{2}}+\frac{\rho_{s}^{2}}{a A_{s}^{2}}-\frac{2 \rho_{p} \rho_{s}}{a A_{\rho} A_{s}}=r_{p}^{2}+\frac{1}{a} r_{s}^{2}-\frac{2}{a} r_{s} r_{p} .
$$

Note that in Fig. 2 the current flowing through the plasma column is the same as that flowing through the nonplasma section. Thus, if $r_{p}<\left|z_{s 2}\right|$, the voltage drop per unit length across the plasma column is less than that across the nonplasma section, and as such the electric field along the surface plasma is lower than that along the nonplasma section. Under this condition, free electrons in the plasma column are dragged outwards by the greater electric field outside the plasma, and so the plasma column may extend into the initially nonplasma region of higher electric field. ${ }^{9}$ Similarly, if $r_{p}>\left|z_{s 2}\right|$, the electric field outside the plasma column is smaller than that along the plasma, and so it cannot drag free electrons in the plasma column outwards, and so drive the surface discharge to elongate. Thus, under the thermal equilibrium condition, the necessary condition for plasma elongation is $r_{p}<\left|z_{s 2}\right|$. As the plasma column elongates when $r_{p}<\left|z_{s 2}\right|$, it gradually takes up the space of the nonplasma section of higher resistance and reduces the overall impedance of the current route, or

$$
\frac{d\left|Z_{T}\right|}{d L_{p}}<0 .
$$

From Eq. (4), the derivative of $\left|Z_{T}\right|^{2}$ with respect to $L_{p}$ is

$$
\begin{aligned}
\frac{d\left|Z_{T}\right|^{2}}{d L_{p}} & =2 v L_{p}-2 \frac{\rho_{s}}{a A_{s}} \frac{\rho_{s}}{A_{s}}-\frac{\rho_{p}}{A_{p}} L \\
& =2 v L_{p}-2 \frac{r_{s}}{a}\left(r_{s}-r_{p}\right) L
\end{aligned}
$$

so the plasma propagation condition of Eq. (6) becomes

$$
\frac{L_{p}}{L}<\frac{r_{s}}{a v}\left(r_{s}-r_{p}\right) \text {. }
$$

Note that the unit length impedance of the nonplasma section satisfies

$$
\begin{aligned}
\left|z_{s 2}\right|=\frac{\left|Z_{s 2}\right|}{L-L_{p}} & =\frac{1}{L-L_{p}}\left|\frac{R_{s} \frac{1}{j \omega C_{s}}}{R_{s}+\frac{1}{j \omega C_{s}}}\right| \\
& =\frac{R_{s}}{L-L_{p}} \frac{1}{\sqrt{1+\left(\omega R_{s} C_{s}\right)^{2}}}=\frac{r_{s}}{\sqrt{a}},
\end{aligned}
$$

thus $r_{p}<\left|z_{s 2}\right|$ implies

$$
r_{p}<r_{s} / \sqrt{a}
$$

Since $a>1$, Eq. (9) suggests that

$$
r_{s}>\sqrt{a} r_{p}>r_{p}
$$

and so the right-hand side of Eq. (8) is always positive.

The maximum plasma length is $L$ when the surface discharge develops into a full-scale flashover arc across the entire width of the capacitor film. So, $0 \leqslant L_{p} / L \leqslant 1$. If, in Eq. (8)

$$
\frac{r_{s}}{a v}\left(r_{s}-r_{p}\right) \geqslant 1,
$$

then the plasma propagation condition of Eq. (8) is always satisfied. It is, therefore, possible for the plasma to develop into a full-scale flashover. With Eq. (5), Eq. (11) becomes

$$
r_{p} \leqslant r_{s} / a
$$

Note that $a=\sqrt{1+\omega^{2} R_{s}^{2} C_{s}^{2}}>1$, so inequality (12) represents a stronger condition for plasma elongation than the condition of Eq. (9). This is the parametric condition under which a surface plasma can, in principle, elongate and develop into a full-scale flashover arc. It should be noted, however, that the plasma resistance changes as it elongates, and so a continuation of plasma elongation requires continued satisfaction of the above condition. A possible scenario when the crosssectional area of the plasma becomes smaller (to maintain approximately the same column volume) is as it elongates. This increases $r_{p}$ according to Eq. (1). Sustained increase in $r_{p}$ can eventually invalidate condition (12) at one point of plasma propagation, the plasma ceases to elongate and is likely to extinguish at this point. On the other hand, if

$$
\frac{r_{s}}{a v}\left(r_{s}-r_{p}\right)<1,
$$

then $L_{p}$ will always be less than $L$. This suggests that the discharge may elongate to a point within the bounds of the electrode film, at which it will extinguish. With the aid of Eq. (9), condition (13) leads to

$$
r_{s} / a<r_{p}<r_{s} / \sqrt{a} .
$$

Inequality (14) specifies the condition under which the surface plasma may propagate but will extinguish before it reaches the electrode boundary. It is of interest to note that plasma elongation conditions (12) and (14) are essentially related to the gradient of the surface electric field at the plasma-surface interface rather than their absolute value. This implies that a surface discharge, and even its elongation, may be maintained by a small surface electric field, much less than that needed to ignite discharge plasmas, as observed, in practice, for outdoor insulators. ${ }^{9,10}$

Although the criteria for plasma elongation under the thermal equilibrium condition are summarized in Eqs. (12) and (14) very simply, their application to practical film capacitors is not straightforward. Data for resistance of surface plasma are not readily available, and so their evaluation is difficult, particularly because plasma resistance also changes as the surface discharge evolves. This difficulty in evaluating plasma resistance prevented a direct assessment of the extent of plasma elongation under the thermal equilibrium condition in our earlier work. ${ }^{8}$ To assess the implications of plasma elongation conditions in Eqs. (12) and (14) to practical film capacitors, we consider dry capacitors (air insulated) at room temperature. For an aluminum-coated electrode with the metallization pattern in Fig. 1, $r_{s}$ is usually between $50-1000 \Omega / \mathrm{cm}^{10,12}$ Suppose electrode segments are $1 \mathrm{~cm}^{2}$ square with a separation gap $0.1 \mathrm{~mm}$ wide and a coating thickness of $10 \mathrm{~nm}$, the maximum value of $C_{s}$ is in the region of $5 \times 10^{-17} \mathrm{~F}$ when there is only one surface gap 
along the nonplasma current route. For a capacitor film 15 cm wide, this suggests that $R_{S} C_{S}$ is, typically, in the order of $10^{-14} \mathrm{~s}$. This requires the source frequency to be around 16 $\mathrm{THz}$ for $\omega R_{s} C_{s}$ to be comparable to unity. As the majority of transient signals encountered in many technological applications are at frequencies well below $100 \mathrm{GHz}$ (microwave frequencies), the effect of the surface capacitance is, therefore, negligible, and so $a \approx 1$. This merges conditions (12) and (14) together to give $r_{p}<r_{s}$, suggesting that the plasma resistance needs to be less than the surface resistance in order for it to elongate. Once $r_{p}<r_{s}$ is satisfied, it is, in principle, possible for an initially small surface plasma to develop into a full-scale flashover arc across the entire width of the capacitor film, and as such potentially lead the film capacitor to fail. However, it is important to note that Eqs. (12) and (14) are necessary but not sufficient conditions for plasma elongation. For instance, if the surface electric field is too weak to supply adequate energy for plasma propagation, plasma elongation, if any, is unlikely to be sustainable even when $r_{p}<r_{s}$ is satisfied.

As an illustration of the likely impact of surface discharges, we consider air plasmas for the case of dry capacitors and we employ the following simple formula of air plasma conductivity in $\Omega^{-1} \mathrm{~cm}^{-1}:^{13}$

$$
\sigma_{p}=1.5 \times 10^{-5} T^{1.5},
$$

and so the unit length plasma resistance is given by

$$
r_{p}=\frac{1}{\sigma_{p}} \frac{1}{\pi d_{p}^{2} / 4}=\frac{8.5 \times 10^{4}}{T^{1.5} d_{p}^{2}},
$$

where $d_{p}$ is the diameter of the plasma column in centimeters and $T$ is its temperature in kelvin. At room temperature, $T \approx 300 \mathrm{~K}$ and for $r_{s}=200 \Omega / \mathrm{cm}$, Eq. (15) leads to

$$
\frac{r_{p}}{r_{s}}=\frac{8.5 \times 10^{4}}{200 \times T^{1.5} d_{p}^{2}}=\frac{0.082}{d_{p}^{2}} .
$$

For the plasma to be more resistive than the surface resistance, so as to prevent any possible subsequent elongation, the initial discharge needs to have a diameter of less than 3 $\mathrm{mm}$. In other words, plasma elongation is possible only when the diameter of the initial discharge is greater than $3 \mathrm{~mm}$. It should be noted that the spacing between two adjacent capacitor film layers (the capacitor winding gap) is usually much less than $3 \mathrm{~mm}$, typically, in the region around $2 \mu \mathrm{m}$, for most modern film capacitors. An initial discharge having a diameter greater than $3 \mathrm{~mm}$ would have to have punctured through more than 136 film layers (usually, each $20 \mu \mathrm{m}$ thick) that sandwich around the surface plasma. This represents a very considerable surface breakdown, a rare event even towards the very end of a film capacitor's life expectancy. In other words, for practical film capacitors of good design, surface discharges with an initial diameter comparable to $3 \mathrm{~mm}$ are very unlikely. This is also consistent with our experience gained from analyzing breakdown damages of striped-down film capacitors after they have been life tested at elevated voltage stressing.

For more realistic surface discharges having an initial diameter less than the capacitor winding gap, say $2 \mu \mathrm{m}$, their resistance is always greater than the surface resistance even at an elevated temperature of $500 \mathrm{~K}$ and an increased surface resistance of $1 \mathrm{k} \Omega$, according to Eq. (16). Consequently, under the thermal equilibrium condition, surface discharges are unlikely to be less resistive than the electrode surface, and as such their elongation on the electrode surface is not electrically encouraged. Hence, in general, after surface plasmas are generated by the locally enhanced surface electric field, they are most likely to shrink in size gradually and eventually extinguish, while depositing their energy thermally to the surrounding capacitor structure. The main effects of surface plasmas are, therefore, to contribute to a possible longterm temperature rise of the film capacitor through the accumulated energy release of collapsed plasmas, and perhaps to microscopic topological changes of the metallization pattern resulting during plasma ignition. In general, these effects are unlikely to affect capacitor performance in any significant manner.

It should be noted, however, that many microdischarges on the electrode surface in practical film capacitors have an initial temperature much higher than the ambient temperature of the capacitor structure. The high temperature of surface plasmas can be an additional source of energy for possible plasma elongation, and as such our above discussion based on the thermal equilibrium may not necessarily include other viable mechanisms for plasma propagation. To this end, the plasma elongation issue needs to be addressed without the thermal equilibrium assumption. This will be discussed in detail in the following section.

\section{IMPACT OF SURFACE PLASMAS UNDER THERMALLY NONEQUILIBRIUM CONDITIONS}

Inequalities (12) and (14) summarize the elongation condition for surface discharge plasmas and their possible evolution into flashover arcs under the thermal equilibrium condition. It is known, however, that breakdown discharges in film capacitors can be much hotter than the plasma containing capacitor structure. Furthermore, the plasma temperature also changes, as the surface plasma propagates on the electrode surface and interacts thermally with its surrounding structure. Hence, it is almost inevitable that there is, in general, a temperature gradient between the plasma column and its surrounding space, especially during plasma ignition. This highlights the need to take into account of thermal effects in our analysis of plasma propagation and its impact on capacitor performance. In this section, we study possible heatconduction-induced mechanisms for plasma elongation.

Surface discharge plasmas are usually ignited by the surface electric field, and subsequently sustained through the surface current. Without heat exchange with its surrounding space, the plasma is heated up through its own Ohmic losses, $i_{p}^{2} R_{p}$, and as such its temperature rises. However, as it interacts thermally with the capacitor structure, its temperature reduces in the form of heat dissipation and radiation into the surrounding capacitor space. Therefore, there are two competing processes for plasma temperature to change. If the net temperature change is negative, the plasma becomes increasingly resistive and as such increasingly unlikely to elongate. 
On the other hand, if the plasma undergoes a positive net temperature change, it becomes increasingly hotter, and as such less resistive, according to Eq. (15). In general, this does not necessarily lead to plasma elongation. However, when the plasma resistance reduces to below the surface resistance of its surrounding electrode area, the discharge plasma begins to elongate, driven by the greater electric field outside its column, as suggested in Eqs. (12) and (14). For cases where the plasma resistance remains larger than the electrode surface resistance throughout the time scale of interest, it is possible for the plasma front (its moving root) to sufficiently heat up the insulating air nearby for the latter to ionize. This air ionization results in a second discharge plasma, which merges with the initial plasma to become a new plasma column of greater length. In other words, this ionization mechanism can lead to an effective plasma elongation. Finally, when there is no net temperature change, the plasma resistance remains unchanged and this represents the critical condition under which plasma elongation is not thermally encouraged.

In general, heat conduction in the plasma-capacitor system is governed by ${ }^{14}$

$$
\rho_{m} c_{p} \frac{\partial T}{\partial t}=k \frac{\partial^{2} T}{\partial x^{2}}+q^{*},
$$

in the one-dimensional limit. Here, $\rho_{m}$ is the mass density of the material under consideration, $c_{p}$ the specific heat, $k$ thermal conductivity, $x$ the distance along the current route from a predefined origin point, and $q^{*}$ the internally generated heat per unit volume. First, we apply the above equation to the plasma column. As an approximation, the thermal radiation loss of the plasma is assumed to be negligible, and so $q^{*}=i_{p}^{2} R_{p} / A_{p} L_{p}$ is the internal heat source for the plasma column. Furthermore, we assume that the $\partial T / \partial t$ term on the left-hand side of Eq. (17) is predominately controlled by $q^{*}$, and so the spatial variation of temperature along the plasma is neglected. Since surface plasmas often spark discharges of short length initially, this is a reasonable approximation. Thus, for the plasma column

$$
\frac{\partial T_{p}}{\partial t}=\frac{i_{p}^{2} r_{p}}{\rho_{m p} c_{p} A_{p}}=g_{1}\left(T_{p}\right) .
$$

The current flowing through the plasma, $i_{p}$, is related to the external voltage $V_{0}$ via the circuit impedance of Eq. (3). As it has been established in the previous section, $a \approx 1$ for frequencies well below $100 \mathrm{GHz}$, and so $Z_{T}=R_{p}+R_{s}$. Consequently,

$$
g_{1}(T)=\frac{E_{0}^{2}}{\rho_{m p}(T) c_{p}(T) A_{p}} \frac{r_{p}(T)}{\left[r_{s}+\left(r_{p}(T)-r_{s}\right)\left(L_{p} / L\right)\right]^{2}},
$$

where $E_{0}=V_{0} / L$ is the average surface electric field.

For the current path along the nonplasma electrode surface, its temperature rise is largely induced by heat conduction from the plasma column while its own Ohmic heating should be, by comparison, negligibly small. In other words, the nonplasma section of the current path acts as a heat sink for the plasma column. Thus, for the nonplasma section Eq. (17) becomes

$$
\rho_{m s} c_{p s} \frac{\partial T_{s}}{\partial t}=k_{s} \frac{f^{2} T_{s}}{f x^{2}} .
$$

Suppose the nonplasma section of length $x_{0}$ has one end, at $x=x_{0}$, attached to the plasma column of temperature $T_{p}$ and its other end, at $x=0$, maintained at the ambient temperature, $T_{\text {amb }}$, of the capacitor structure. Equation (20) can be solved analytically, ${ }^{14}$ and one solution is

$$
T_{s}(x, t)=T_{\mathrm{amb}}+\left(T_{p}(t)-T_{\mathrm{amb}}\right) \sin \frac{\pi x}{2 x_{0}} e^{-\left(\pi / 2 x_{0}\right)^{2} \alpha_{s} t},
$$

where $\alpha_{s}=k_{s} / \rho_{m s} c_{p s}$ is the thermal diffusivity of the electrode material. At the plasma interface with the nonplasma section, the temperature is reduced according to

$$
T_{s}\left(x_{0}, t\right)=T_{\mathrm{amb}}+\left(T_{p 0}-T_{\mathrm{amb}}\right) e^{-\left(\pi / 2 x_{0}\right)^{2} \alpha_{s} t},
$$

where $T_{p 0}$ is the plasma temperature $t=0$ and also the temperature of the nonplasma section at its interface with the plasma and at $t=0$.

For many film capacitors used in power systems and other technological applications, their electrode material is either aluminum or its alloy. At room temperature, $\alpha_{s}$ $=0.06-0.08 \mathrm{~m}^{2} / \mathrm{s}$ for pure aluminum and some of its alloy forms. ${ }^{14}$ If we choose $x_{0}$ to be approximately the half width of the capacitor electrode, say, $8 \mathrm{~cm}$ (implying a very significantly elongated plasma $7 \mathrm{~cm}$ long on a $15-\mathrm{cm}$ wide capacitor film), then $\left(\pi / 2 x_{0}\right)^{2} \alpha_{s} t \approx 30 t$. As a single gas discharge event usually lasts well within the millisecond range in film capacitors, the above term is very small, and so

$$
T_{s}\left(x_{0}, t\right)-T_{p 0} \approx-\left[T_{p 0}-T_{\mathrm{amb}}\right]\left(\pi / 2 x_{0}\right)^{2} \alpha_{s} t .
$$

It should be noted that for discharge plasmas of much shorter initial length, $x_{0}$ is larger than $8 \mathrm{~cm}$, and as such $\left(\pi / 2 x_{0}\right)^{2} \alpha_{s} t$ is even smaller.

When the temperature reduction of the plasma column due to the heat conduction through its moving root [Eq. (23)] is the same as its temperature rise characterized in Eq. (18), the plasma will maintain its initial temperature. Under this condition, it is unlikely for the plasma to become less resistive, according to Eq. (15), and as such it will not elongate unless dragged by a greater electric field outside the plasma column. In other words, a surface plasma is unlikely to undergo a thermally driven elongation when its temperature rise due to Ohmic heating reduces to the same as its temperature drop due to heat conduction. To this end, we introduce the ratio of temperature change

$$
f(T) \equiv \frac{\left|\Delta T_{\text {rise }}\right|}{\left|\Delta T_{\text {drop }}\right|}=\frac{g_{1}(T)}{\left(T-T_{\text {amb }}\right)\left(\pi / 2 x_{0}\right)^{2} \alpha_{s}},
$$

such that $f(T)=1$ represents the above-mentioned critical condition. Note that $x_{0}=L-L_{p}$, and so Eq. (24) reduces to

$$
f(T)=\frac{\left(1-L_{p} / L\right)^{2}}{\left[1+\left(r_{p} / r_{s}-1\right) L_{p} / L\right]} \Theta(T),
$$




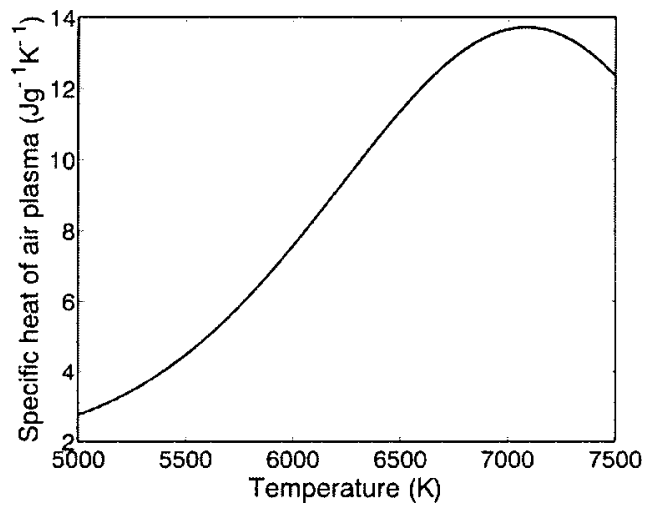

FIG. 3. Specific heat of air plasma as a function of temperature.

where

$$
\Theta(T)=\frac{L^{2}}{A_{p}} \frac{4}{\pi^{2}} \frac{E_{0}^{2} r_{p} / r_{s}^{2}}{c_{p} \rho_{m p} \alpha_{s}\left(T-T_{\mathrm{amb}}\right)} .
$$

The above equation is a measure of the relative temperature increase of the plasma column in the one-dimensional limit, in which the plasma column is assumed to have the same cross-sectional area as the film capacitor. As the initial surface spark discharge is usually short and filamentary, this is an overestimate of the thermal impact of the plasma. To account for this, the plasma temperature rise of Eq. (18) is reduced by the ratio of the cross-sectional area of the plasma column, $A_{p}$, and that of a film capacitor, $d_{c} \times L_{w}$, with $d_{c}$ being the thickness of the plasma containing capacitor pack and $L_{w}$ its length. Furthermore, as the lateral heat conduction of the plasma is also possible cross the plasma column, an additional coefficient of $d_{p} L_{p} / d_{c} L$ should be introduced. Assuming $L_{w}=L$ for a square capacitor pack, Eq. (26) becomes

$$
\Theta(T)=\frac{4}{\pi^{2}} \frac{d_{p} L_{p}}{d_{c}^{2}} \frac{E_{0}^{2} r_{p} / r_{s}^{2}}{c_{p} \rho_{m p} \alpha_{s}\left(T-T_{\mathrm{amb}}\right)} .
$$

If $f(T)>1$, the temperature rise of the plasma column cannot be compensated by the temperature reduction due to its heat conduction to the nonplasma region. As a result, the plasma becomes hotter and less resistive. This can lead to its elongation into its surrounding space when it becomes less resistive than the electrode surface. If $f(T)<1$, the temperature rise of the plasma column is well offset by the temperature

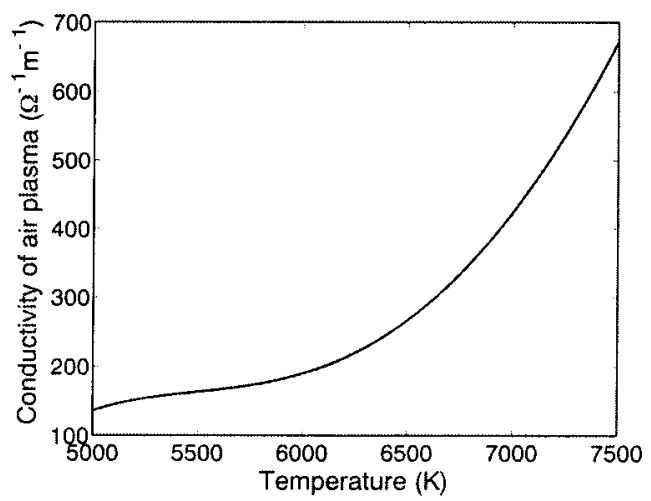

FIG. 4. Conductivity of air plasma as a function of temperature.

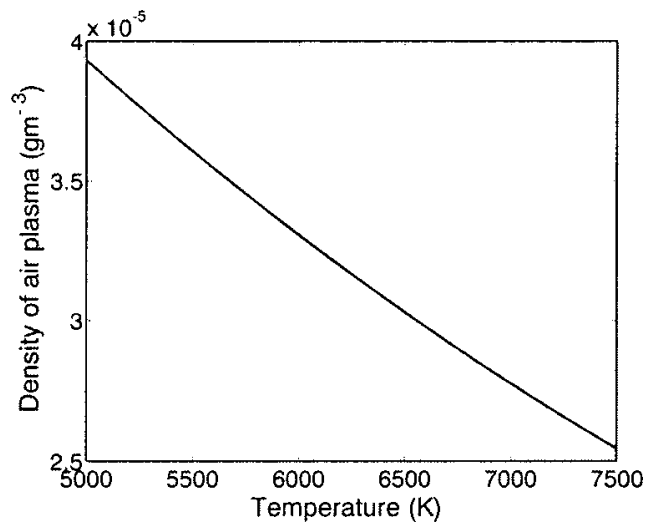

FIG. 5. Density of air plasma as a function of temperature.

reduction of the nonplasma area, and as such the plasma becomes progressively colder and eventually extinguished.

To illustrate whether a surface plasma may elongate under thermally nonequilibrium conditions, we consider a film capacitor with $r_{s}=200 \Omega / \mathrm{cm}$ and $T_{\mathrm{amb}}=298 \mathrm{~K}$. For practical multiple-layer film capacitors, $d_{c}=2-5 \mathrm{~cm}$, and so we choose $d_{c}=2.5 \mathrm{~cm}$ for all cases discussed here. We now assume that the surface discharge is an air plasma with a diameter of $d_{p}=2 \mu \mathrm{m}$ and employ Eq. (27) to identify the parametric condition under which the air plasma may undergo a net temperature rise. The specific heat of the air plasma is obtained from Ref. 15 and plotted in Fig. 3 as a function of temperature at one atmospheric pressure. Similarly, electrical conductivity of the air plasma and its density are plotted against the temperature in Figs. 4 and 5 based on the data in Refs. 16 and 17, respectively. For the electrode surface, the thermal diffusivity of aluminum is shown in Fig. $6{ }^{18}$ It is important to mention that we deliberately choose a very high temperature for surface plasmas in an attempt to assess the worst scenario in which the film capacitor is impacted on by the most severe and hottest possible plasmas. However, one implication is that the use of the data shown in Figs. 3-6 and Eq. (27) does not permit a detailed description of the dynamics of more realistic, but much milder, surface plasmas over a more probable temperature range below 5000 K. Nevertheless, these less-severe plasmas should have a much smaller impact on capacitor performance. Given that

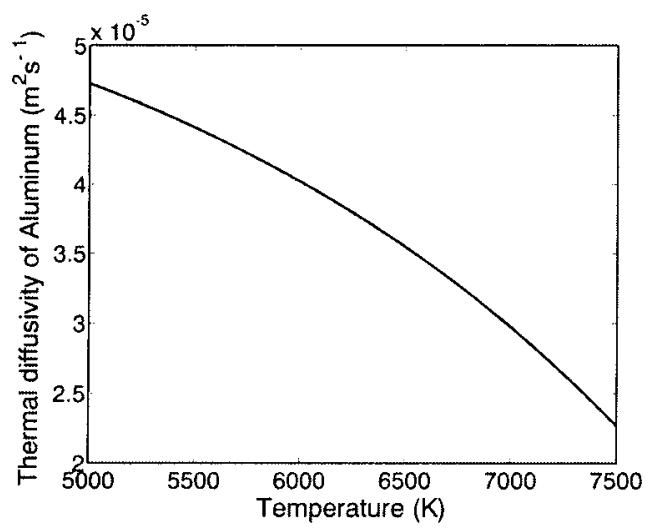

FIG. 6. Thermal diffusivity of aluminum as a function of temperature (see Ref. 18). 


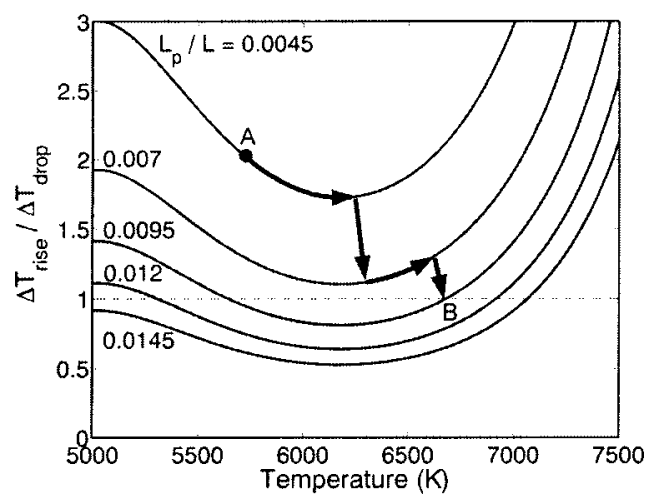

FIG. 7. Normalized temperature rise of an air plasma as a function of its initial temperature at different initial plasma length.

our prime objective is to understand whether surface discharges may affect significantly capacitor performance under any possible circumstances, it is more profitable to assess the worst scenario caused by the most severe plasmas first before the detailed dynamic features of much milder and realistic surface plasmas are unraveled.

It is clear from Eq. (27) that heat transfer between the plasma column and its surrounding structure depends very much upon the surface electric field, which drives the surface current to provide the necessary electric energy for plasma evolution. In our previous work, we considered plasma propagation at a surface field of $200 \mathrm{~V} / \mathrm{cm},{ }^{8}$ since this is illustrative of the level of surface electric field that may cause sustained plasma elongation in outdoor insulators. ${ }^{9}$ At this level of surface electric field, a surface discharge may elongate to between $30 \%$ and $40 \%$ of the width of a film capacitor. This is a very significant plasma elongation and it would cause considerable damage to film capacitors. A more accurate evaluation of the surface electric field may be obtained using an equivalent circuit model developed recently for film capacitors. ${ }^{10}$ It has been shown that for ac capacitors at $15 \mathrm{kHz}$ or below, the rms value of the surface electric field is, at most, $20 \mathrm{~V} / \mathrm{cm} .{ }^{10}$ A higher surface electric field can be achieved at higher frequencies, but the very short period of these high-frequency signals is such that they may not be able to support a surface discharge throughout the entire duration of its development. In other words, surface discharges are likely to experience an effective electric field that is much less than predicted for frequencies much greater than $15 \mathrm{kHz}$. To this end, we assume that the surface electric field is a constant at $20 \mathrm{~V} / \mathrm{cm}$.

Based on the parameters chosen above and Eqs. (25) and (27), the normalized temperature rise of the plasma column is plotted in Fig. 7 as a function of its initial temperature using the data in Figs. 3-6. Suppose a surface discharge is initially $0.0045 \mathrm{~L}$ long $(0.675 \mathrm{~mm}$ on a 15 -cm-wide capacitor film) at about $5700 \mathrm{~K}$, corresponding to a plasma operation point at point $A$ on the top curve in Fig. 7. Since there is a positive net temperature rise at point $A$, the plasma temperature goes up subsequently and its operation point moves along the top curve towards the right to the highertemperature region in Fig. 7. As the plasma becomes hotter and hotter, it may start to elongate through either an electric- field gradient at its boundary with the nonplasma section or an amalgamation with an additional ionized air induced around its moving root. In Fig. 7, this corresponds to the plasma operation point as it moves to a lower curve having a greater $L_{p} / L$ ratio (thus, longer plasma length), for instance, the $L_{p} / L=0.007$ curve. In practice, this process consumes a sizable amount of plasma energy prior to its elongation, and so imposes a restriction on the extent of future plasma elongation. If the new operating point of the discharge plasma still has a positive net temperature rise, the plasma temperature continues to increase until further elongation processes move the plasma to an operation point with a zero net temperature rise such as point $B$ in Fig. 7. With a surface electric field at $20 \mathrm{~V} / \mathrm{cm}$, the plasma column at point $B$ in Fig. 7 would be maintained around $6640 \mathrm{~K}$ and $0.0095 \mathrm{~L}(1.425$ $\mathrm{mm}$ on a $15-\mathrm{cm}$-wide capacitor film). Given that one electrode element is about $1 \mathrm{~cm}$ wide by $1 \mathrm{~cm}$ long, such a steady-state plasma would be unlikely to cause any significant damage to the film capacitors. In practice, a constant surface field can very rarely be maintained forever. When, and as, the surface field reduces, the plasma will be gradually absorbed thermally into its surrounding structure and eventually extinguished.

For surface discharges with a different initial length from $0.0045 L$, they are likely to undergo a similar process to the $A \rightarrow B$ route in Fig. 7, provided they experience initially a net temperature rise. Specifically, the discharge plasma is likely to undergo either a field-gradient-induced elongation [conditions (12) and (14)] or gas-ionization-induced elongation such that it settles at an equilibrium point like point $B$ in Fig. 7 before being absorbed thermally. It is worth noting that all the curves in Fig. 7 show an upturn in net temperature rise above around $6200 \mathrm{~K}$. For cases where the discharge plasma does not elongate in the time scale of interest, this does not mean an unrestricted temperature rise for the plasma. In practice, the surface electric field reduces from its nominal peak value, at least periodically as in ac applications, and as such the electric energy required to drive up the plasma temperature withdraws and the hot plasma starts to be absorbed thermally by its surrounding structure. Since gas discharge events last well within $1 \mathrm{~ms}$, it is highly probable that the discharge plasma will extinguish before the withdrawn electric energy is re-fed into the discharge region.

For surface plasmas with a negative net temperature rise initially, such as those on the $L_{p} / L=0.0145$ curve at a temperature below $7000 \mathrm{~K}$ in Fig. 7, they become increasingly colder and less resistive. Thus, it is unlikely for them to elongate subsequently. Instead, they shrink in size gradually and extinguish eventually. From the above discussion of Fig. 7 , it appears that there are always mechanisms for surface discharges of varying initial conditions to extinguish at an eventual length much smaller than the width of the capacitor film. Hence, surface breakdowns and their discharge plasmas are not very likely to evolve into significant flashover arcs for the system parameters used in Fig. 7. At a greater surface electric field or a larger plasma diameter, however, the length at which the plasma extinguishes is found numerically to be at a larger proportion of the capacitor film width. Therefore, there is a theoretical possibility for a surface discharge to 


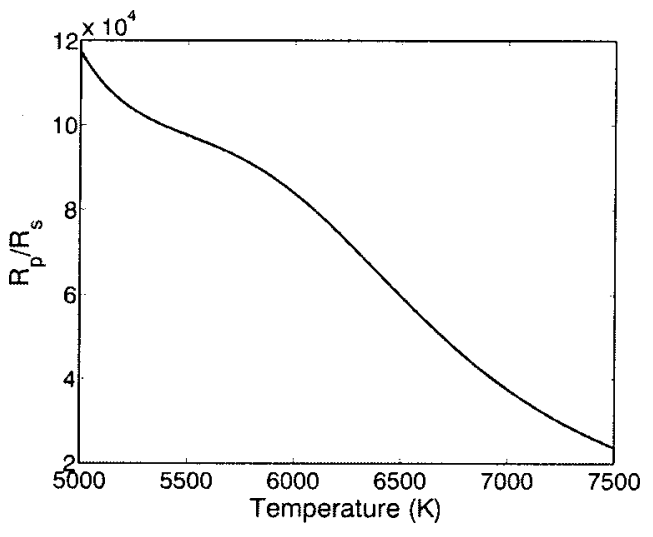

FIG. 8. Normalized plasma resistance as a function of plasma temperature.

have a momentary tendency to evolve into a significant flashover arc. However, as already illustrated, discharge plasmas are not likely to be supported permanently at a constant peak surface field and a periodic reduction in the latter reduces considerably the impact of possible plasma elongation. As the capacitor winding gap is around $2 \mu \mathrm{m}$, surface plasmas with a diameter much greater than $2 \mu \mathrm{m}$ are also unlikely. Equally important is the fact that we deliberately choose a temperature range that is well above what can be normally expected for surface discharges in film capacitors in an attempt to assess the maximum possible impact of surface discharges and their elongation. Therefore, the plasma elongation illustrated by Fig. 7 is a considerable overestimate, and as such the general conclusion of insignificant effects of surface discharges on capacitor performance should remain valid even at a greater surface electric field.

It is also of interest to note that the plasma resistance used in Fig. 7 is much higher than the surface resistance, as shown in Fig. 8. It is clear that for the example discussed in Fig. 7 plasma elongation due to the electric-field gradient is not possible. Instead, the plasma elongation indicated in Fig. 7 is entirely due to the heat conduction of the plasma column. In this case, the significant temperature gradient between the plasma column and its surrounding space results in an overwhelming heat-conduction effect on the plasma propagation and the electric-field-based argument of Eq. (6) becomes irrelevant. For surface discharges, in general, there are generically two mechanisms for plasma elongation, namely, the electric-field gradient and the temperature gradient. For practical film capacitors, these two mechanisms may exist simultaneously and the detailed processes of plasma propagation are likely more complicated than described in the formulation developed in this study. However, these detailed features are of secondary importance, as designers and users of film capacitors are predominately concerned with whether surface plasmas may reduce significantly capacitor life and reliability. From our physical model that deliberately exaggerates the impact of these surface plasmas, it seems that surface plasmas should not affect capacitor performance significantly. Thus, even when more sophisticated plasma models are developed in the future to unravel the detailed dynamic features of surface discharges, they are unlikely to change the general conclusion that surface plasmas have an insignificant impact on capacitor performance.

\section{CONCLUSION}

We have developed two simplified analytical formulations to study propagation and elongation of surface breakdown plasmas in metallized film capacitors under thermal equilibrium conditions and thermally nonequilibrium conditions, respectively. A surface field gradient mechanism was used to characterize the propagation of cold surface plasmas, whereas a temperature gradient mechanism was proposed to capture the essential features of the hot surface plasmas when propagating and elongating on the electrode surface. Numerical examples were then used to assess the worse scenario whereby the film capacitors are impacted on by large surface plasmas of the greatest possible diameter, at the highest possible temperature, driven by a significant surface electric field. From our studies of these worse cases, it has been shown that while surface breakdown discharges may elongate they are unlikely to evolve into significant flashover arcs. Therefore, their impact on capacitor performance is likely to be insignificant. This finding is consistent with our observation from discharge damage analyses of aged film capacitors.

It should be mentioned, however, that our discussion is based on the square metallization pattern of Fig. 1, for which surface resistance is known and performance of relevant practical film capacitors is also known. For film capacitors of radically different metallization patterns, their surface resistance can be much greater than assumed for that in Fig. 1 and their induced surface field intensification may also be significant such that the surface field is in excess of $20 \mathrm{~V} / \mathrm{cm}$ for frequencies below $15 \mathrm{kHz}$. As a result, the impact of surface breakdown discharges needs to be reassessed for these different film capacitors using the theoretical models developed in this study.

\section{ACKNOWLEDGMENTS}

One of the authors (Y.P.L.) acknowledges financial support from ABB Power T \& D, Capacitor Division, UK, and Loughborough University, and the CVCP committee (UK).

${ }^{1}$ D. G. Shaw, S. W. Cichanowski, and A. Yializis, IEEE Trans. Electr. Insul. 16, 399 (1981).

${ }^{2}$ C. W. Reed and S. W. Chichanowski, IEEE Trans. Dielectr. Electr. Insul. 1, 904 (1994).

${ }^{3}$ B. Sanden and E. Ildstad, Proceedings of the 1998 IEEE International Conference on Conduction and Breakdown in Solid Dielectrics, Vasteras, Sweden, June (1998), pp. 210-213 (unpublished).

${ }^{4}$ A. Gadoum, B. Gosse, and J.-P. Gosse, IEEE Trans. Dielectr. Electr. Insul. 2, 1075 (1995).

${ }^{5}$ W. Khachen and J. R. Laghari, IEEE Trans. Electr. Insul. 27, 1022 (1992).

${ }^{6}$ A. Schneuwly, P. Groning, L. Schlapbach, C. Irrgang, and J. Vogt, IEEE Trans. Dielectr. Electr. Insul. 5, 862 (1998).

${ }^{7}$ J. Kammermaier, G. Rittmayer, and S. Birkle, J. Appl. Phys. 66, 1594 (1989).

${ }^{8}$ M. G. Kong and Y. P. Lee, Proceedings of the IEEE International Conference on Dielectrics and Electrical Insulation Phenomenon, Victoria, BC, Canada, October (2000) (unpublished).

${ }^{9}$ H. Boehme and F. Obenaus, CIGRE Paper 407 (1966) (unpublished). 
${ }^{10}$ Y. P. Lee, M. G. Kong, M. R. Dunn, and D. Young-Cannon, Proceedings of the IEEE International Conference on Dielectrics and Electrical Insulation Phenomena, Victoria, BC, Canada, October (2000) (unpublished).

${ }^{11}$ N. Dhahbi-Megriche, A. Beroual, and L. Krahenbuhl, J. Phys. D 30, 889 (1997).

${ }^{12}$ G. J. Walters (private communications).

${ }^{13}$ I. Fofana and A. Beroual, J. Phys. D 30, 1653 (1997).

${ }^{14}$ A. J. Chapman, Heat Transfer, 3rd ed. (MacMillan, New York, 1974).

${ }^{15}$ R. N. Gupita, K. P. Lee, R. A. Thompson, and J. M. Yos, "Calculations and curve fits of thermodynamic and transport properties for equilibrium air to 30000 K," NASA Reference Publication No. 1260 (1991).

${ }^{16}$ S. V. Dresvin, Physics and Technology of Low-Temperature Plasmas (The Iowa State University Press, Ames, IA, 1977).

${ }^{17} \mathrm{H}$. Kroepelin et al., Thermodynamic Diagrams for High Temperature Plasmas of Air, Air-Carbon, Carbon-Hydrogen Mixtures, and Argon (Pergamon, Oxford, 1971).

${ }^{18}$ Y. S. Touloukian, R. W. Powell, C. Y. Ho, and M. C. Nicolaou, Thermophysical Properties of Matter, Volume 10: Thermal Diffusivity (1973). 